\title{
Addressing the Long-term Impact of ML Decisions via Policy Regret
}

\author{
David Lindner $^{1}$, Hoda Heidari ${ }^{2}$, Andreas Krause ${ }^{1}$ \\ ${ }^{1}$ ETH Zurich \\ ${ }^{2}$ Carnegie Mellon University \\ \{lindnerd,krausea\}@ethz.ch,hheidari@cmu.edu
}

\begin{abstract}
Machine Learning (ML) increasingly informs the allocation of opportunities to individuals and communities in areas such as lending, education, employment, and beyond. Such decisions often impact their subjects' future characteristics and capabilities in an a priori unknown fashion. The decision-maker, therefore, faces explorationexploitation dilemmas akin to those in multi-armed bandits. Following prior work, we model communities as arms. To capture the long-term effects of ML-based allocation decisions, we study a setting in which the reward from each arm evolves every time the decision-maker pulls that arm. We focus on reward functions that are initially increasing in the number of pulls but may become (and remain) decreasing after a certain point. We argue that an acceptable sequential allocation of opportunities must take an arm's potential for growth into account. We capture these considerations through the notion of policy regret, a much stronger notion than the often-studied external regret, and present an algorithm with provably sub-linear policy regret for sufficiently long time horizons. We empirically compare our algorithm with several baselines and find that it consistently outperforms them, in particular for long time horizons.
\end{abstract}

\section{Introduction}

Machine learning (ML) systems increasingly inform or make high-stakes decisions about people, in areas such as credit lending [Dobbie et al., 2018], education [Marcinkowski et al., 2020], criminal justice [Berk and Hyatt, 2015], employment [Sánchez-Monedero et al., 2020], and beyond. These ML-based decisions can negatively impact alreadydisadvantaged individuals and communities [Sweeney, 2013; Buolamwini and Gebru, 2018; Angwin et al., 2016]. This realization has spawned an active area of research into quantifying and mitigating the disparate effects of ML [Dwork et al., 2012; Kleinberg et al., 2017; Hardt et al., 2016]. Much of this work has focused on the immediate predictive disparities that arise when supervised learning techniques are applied to batches of training data sampled from a fixed un- derlying population [Dwork et al., 2012; Zafar et al., 2017; Hardt et al., 2016; Kleinberg et al., 2017]. While such approaches capture important types of disparity, they fail to account for the long-term effects of present decisions on individuals and communities. Recent work has advocated for shifting the focus to societal-level implications of ML in the long run [Liu et al., 2018; Hu et al., 2019; Heidari et al., 2019; Dong et al., 2018; Milli et al., 2019].

In many real-world domains, decisions made today correspond to the allocation of opportunities and resources that impact the recipients' future characteristics and capabilities. In such settings, we argue that a socially and ethically acceptable allocation of opportunities must account for the recipients' long-term potential for turning resources into social utility. As an example, consider the following stylized scenario: Suppose a decision-maker must allocate funds to several communities, all residing in one city, at the beginning of every fiscal period. The communities have distinct racial and wealth compositions, and for historical reasons, they initially have different capabilities to turn their allocated funds into economic prosperity and welfare for members of the community and the city. The decision-maker does not know ahead of time how the economic capabilities of each community will evolve in response to the funds allocated to it. Moreover, he/she can only observe the return on each possible allocation strategy after employing it. While the decision-maker does not know the precise return-on-investment or reward curves associated with each community in advance, domain knowledge may provide him/her with information about the general shape of such curves. For instance, he/she may be able to reliably assume that reward curves are often initially increasing with diminishing marginal returns; and if investment continues beyond a point of saturation, they exhibit decreasing returns to additional investments.

How should a just-minded decision-maker allocate funds in this hypothetical example? Should he/she always aim for equal allocation of funds in every fiscal period to ensure a form of distributive equality today, or are there cases ${ }^{1}$ in which he/she should additionally take each community's potential for growth into account and allocate funds proportionately? Note that in this example, a myopic decision

\footnotetext{
${ }^{1}$ For example, such considerations may come to the fore once all communities have received a reasonable minimum budget.
} 
maker might neglect disadvantaged communities with high long-term potential to turn funds into welfare, and as a result, amplify disparities between advantaged and disadvantaged communities over time. If the decision-maker aims to maximize the city's long-term economic welfare and prosperity, he/she should prioritize communities that produce higher returns on investment over time. Aside from the utilitarian argument for this objective, it can also be justified through the classic fitness argument to justice and fairness, which states that resources and opportunities must be allocated to those who make the best use of them [Sandel, 2010; Moulin, 2004]. ${ }^{2}$ This objective motivates the algorithmic question we focus on in this work: how should the decisionmaker choose a sequence of allocations to ensure that communities receive funds in proportion to their relative potential for producing high reward for society in the long-run?

Motivated by the above example and numerous other realworld domains in which similar concerns arise, ${ }^{3}$ we study a multi-armed-bandit (MAB) setting in which communities correspond to arms and the reward from each arm evolves every time the decision-maker pulls that arm. We consider a decision-maker who aims to maximize the overall reward obtained within a set time-horizon, but because he/she does not know how the reward curves evolve, he/she is bound to incur some regret. We formulate the decision-maker's goal in this sequential setting as achieving low policy regret [Arora et al., 2012]. As Figure 1 shows, conventional no external regret algorithms ignore the impact of their decisions today on the evolution of rewards, so they are prone to spending many of their initial pulls on arms that exhibit high immediate rewards but lack adequate potential for growth.

Technical findings. We study single-peaked bandits, a new MAB setting with reward functions that are initially increasing and concave in the number of pulls but can become decreasing at some point (Section 2). We introduce SinglePeaked Optimism (SP0), a novel algorithm that considers potential long-term effects of pulling different arms (Section 3). We prove that SPO achieves sub-linear policy regret if rewards can be observed free of noise (Section 3.1). Further, we present an LP-based heuristic that effectively handles noisy reward observations (Section 4). We empirically compare SPO with several standard no-external-regret algorithms and additional baselines, and find that SPO consistently performs better, in particular, for long time horizons (Section 5).

Broader implications. Our work takes conceptual and technical steps toward modeling and analyzing the long-term

\footnotetext{
${ }^{2}$ We emphasize that in many domains, considerations such as need and rights should take precedence to fitness as defined in our stylized example. In certain domains, however, fitness can be one of the key criteria in determining whether an allocation is morally acceptable. For the sake of simplicity and concreteness, we solely focus on this particular factor. It is worth noting that our model and findings are equally applicable to settings in which needs or entitlements change in response to the sequence of allocation decisions.

${ }^{3}$ Additional real-world examples that fit into our model include: allocating policing resources to neighborhoods to maximize safety; allocating funds to research institutions to maximize scientific discoveries and innovations; allocating loans to students to maximize the rate of graduation/ loan pay-back.
}
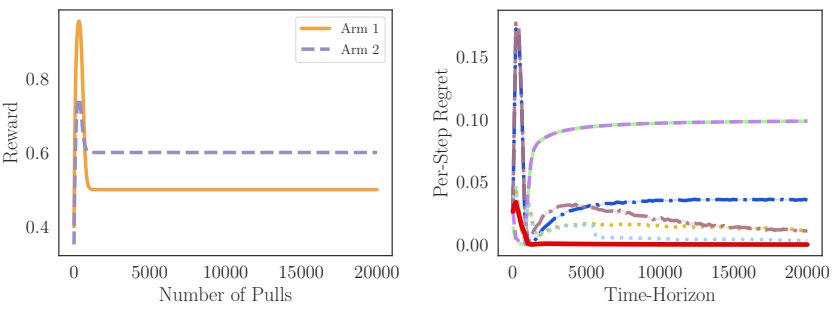

Figure 1: The left plot shows a single-peaked bandit with two reward functions, modeling the evolution of rewards in the number of times each arm is pulled. For long time horizons, the optimal strategy is to play Arm 2 because it has a higher asymptotic reward. However, bandit algorithms that maximize external regret fail to recognize this because the initial reward of Arm 2 is smaller than the initial and asymptotic reward of Arm 1. The right plot shows the regret of a greedy-selection strategy $(--)$, EXP3 [Auer et al., 2002] (- ), which minimizes external regret, as well as DUCB [Garivier and Moulines, 2011] (" " " ), SW-UCB [Garivier and Moulines, 2011] ( * "), and R-EXP3 [Auer et al., 2002] (-), three bandit algorithms designed for nonstationary bandits. All of these algorithms fail on the single-peaked bandit. We propose SPO (-) which achieves sub-linear policy regret in single-peaked bandits settings. For more details on our experiments, see Section 5.

implications of ML-informed allocations made over time. From a conceptual point of view, our work showcases the importance of accounting for domain knowledge (here, the general shape of reward curves) and social-scientific insights (e.g., the dynamic by which communities evolve in response to allocation policies) to formulate ML's long-term impact. Our results draw attention to the necessity of understanding social dynamics of a domain for designing allocation algorithms that improve equity and fairness in the long-run. From a technical perspective, we believe our work can serve as a stepping stone toward designing and analyzing better nopolicy regret algorithms for domain-specific reward curves beyond those considered here. Our work is directly applicable to a specific class of reward functions which generalizes and subsumes those in prior work (e.g., [Heidari et al., 2016]). Finally, our novel approach to handling noise allows utilizing the proposed algorithm in more practical settings where observed rewards are expected to be noisy.

\subsection{Related Work}

Much of the existing work on the social implications of ML focuses on disparities in a model's predictions [Dwork et al., 2012; Zafar et al., 2017; Hardt et al., 2016; Kleinberg et al., 2017]. However, these approaches are only suited to evaluate one-shot decision scenarios. In contrast, we formalize disparities that arise when making a sequence of allocation decisions. Recent work has initiated the study of longer-term consequences and effects of MLbased decisions on people, communities, and society. For example, [Liu et al., 2018] and [Kannan et al., 2019] study how a utility-maximizing decision-maker may interpret and use ML-based predictions. [Dong et al., 2018], [Hu et al., 2019], and [Milli et al., 2019] address strategic classification, a setting in which decision subjects are assumed to respond strategically and untruthfully to the choice of the classifica- 
tion model, and the goal is to design classifiers that are robust to strategic manipulation. [Hu and Chen, 2018] study the impact of enforcing statistical parity on hiring decisions made in a temporary labor market that precedes a permanent labor market. [Mouzannar et al., 2019] and [Heidari et al., 2019] model the dynamics of how members of a population react to a selection rule by changing their qualifications (defined in terms of true labels or feature vectors). However, none of the prior articles investigate the community-level implications of ML-based decision-making policies over multiple time-steps.

Another conceptually-relevant line of work studies fairness in online learning [Joseph et al., 2016; Joseph et al., 2017; Jabbari et al., 2017]. [Joseph et al., 2016], for example, study fairness in the MAB setting, where arms correspond to socially salient groups (e.g., racial groups), and pulling an arm is equivalent to choosing that group (e.g., to allocate a loan to). They consider an algorithm fair if it never prefers a worse arm to a better one, that is, the arm chosen by the algorithm never has a lower expected reward than the other arms. Similar to [Joseph et al., 2016], in our running example, each arm corresponds to a community. However, instead of imposing short-term notions of fairness, we focus on longer-term implications and disparities arising from present decisions.

Our model is based on the MAB framework, which has been established as a powerful tool for modeling sequential decision-making, and has been used successfully for many decades and across a wide range of real-world domains [Gittins et al., 2011; Bubeck et al., 2012]. From a technical perspective we study a nonstationary bandit problem. In nonstationary bandits (with limits to the change of the reward distributions), modified versions of common bandit algorithms have strong theoretical guarantees and good empirical performance. For example, if the reward distributions only change a small number of times, variants of the upper confidence bound algorithm (UCB) such as discounted or sliding-window UCB perform well [Garivier and Moulines, 2011]. Similarly, if there is a fixed budget on how much the rewards can change, R-EXP3, a variant of the popular EXP3 algorithm for adversarial bandits [Auer et al., 2002], guarantees low regret [Besbes et al., 2019]. In this work, we do not restrict how much the rewards can change, but instead restrict the functional shape of the reward functions to be first increasing and concave before switching to decreasing. This is somewhat similar to rotting bandits [Levine et al., 2017] and recharging bandits [Kleinberg and Immorlica, 2018], but distinct from them in crucial ways. In contrast to rotting bandits, where the rewards decrease when pulling an arm more often, our reward functions first increase and only later decrease with the number of pulls. In contrast to recharging bandits, where rewards are increasing and concave in the amount of time an arm has not been pulled, we consider a bandit setting with rewards depending on the number of times an arm has been pulled. We consider reward functions that exhibit a "unimodal" shape. However, our setting is very different from unimodal bandits, which are stationary bandit models with a unimodal structure across arms [Yu and Mannor, 2011; Combes and Proutiere, 2014].

The setting by [Heidari et al., 2016] is closest to ours. They consider two separate models, one with rewards that are in- creasing and concave, and another with decreasing rewards in the number of pulls of an arm. While [Heidari et al., 2016] provides different algorithms for these two cases, we present a single algorithm that can adapt to both settings and beyond, while matching the respective asymptotic policy regret bounds in [Heidari et al., 2016] (cf. Appendix B ${ }^{4}$ ). Additionally, in contrast to [Heidari et al., 2016] who primarily study noise-free observations, we provide an effective heuristic for handling noise.

For an extended discussion of prior work, see Appendix A.

\section{The Single-Peaked Bandit Setting}

We consider a multi-armed bandit (MAB) with arms $\{1, \ldots, N\}$, corresponding, e.g., to the different communities in our introductory example. At each time step $t=$ $1,2, \ldots T$, the decision-maker pulls one arm and observes its immediate reward, e.g., the short-term outcome of an investment in a community. The decision-maker aims to achieve the highest cumulative reward within the fixed time horizon $T$, e.g., he/she wants to get the best total return-on-investment over $T$ years. Each arm $i$ has an underlying reward function $f_{i}:\{1, \ldots, T\} \rightarrow[0,1]$. When the decision-maker pulls arm $i$ for the $m$-th time $(1 \leq m \leq T)$, he/she observes reward $f_{i}(m)$. Later, in Section 4 , we study noisy reward observations of the form $f_{i}(m)+\epsilon_{i}$, but for now, let's assume observed rewards are noise-free. We denote the cumulative reward of arm $i$ after $m$ pulls by $F_{i}(m)=\sum_{t=1}^{m} f_{i}(t)$.

A deterministic policy $\pi$ is a sequence of mappings $\left(\pi_{1}, \ldots, \pi_{T}\right)$ from observed action-reward histories to arms, where $\pi_{t}$ maps histories of length $(t-1)$ to the next arm to be pulled:

$$
\pi_{t}:\{1,2, \ldots, N\}^{t-1} \times[0,1]^{t-1} \rightarrow\{1,2, \ldots, N\} .
$$

The cumulative reward of a policy only depends on how often it pulls each arm, so it is determined by a tuple $\left(n_{1}^{T}(\pi), \ldots, n_{N}^{T}(\pi)\right)$ where $n_{i}^{T}(\pi)$ denotes how often $\pi$ pulls $\operatorname{arm} i$ within the time horizon $T$. Note that $\sum_{i=1}^{N} n_{i}^{T}(\pi)=T$. We can write the cumulative reward of a policy as:

$$
r_{T}(\pi)=\sum_{i=1}^{N} \sum_{t=1}^{n_{i}^{T}(\pi)} f_{i}(t)=\sum_{i=1}^{N} F_{i}\left(n_{i}^{T}(\pi)\right) .
$$

Let $\Pi$ denote the space of all possible deterministic policies, and OPT $\in \operatorname{argmax}_{\pi \in \Pi} r_{T}(\pi)$ be an optimal policy, that is, a policy achieving the highest possible cumulative reward. The decision-maker does not know the reward functions $\left(f_{i}\right.$ 's) in advance, so he/she cannot find an optimal policy ahead of time. Instead, he/she can aim to design a (possibly stochastic) policy that minimizes the policy regret: $r_{T}(\mathrm{OPT})-\mathbb{E} r_{T}(\pi)$. Given a fixed set of reward functions, we say an algorithm $\mathcal{A}$ that follows policy $\pi^{\mathcal{A}, T}$ over time horizon $T$ has sub-linear policy regret, if

$$
\lim _{T \rightarrow \infty} \frac{r_{T}(\mathrm{OPT})-\mathbb{E} r_{T}\left(\pi^{\mathcal{A}, T}\right)}{T}=0 .
$$

${ }^{4}$ All appendicies can be found in the extended paper at: https://arxiv.org/abs/2106.01325. 
It is in general impossible to achieve sub-linear policy regret in an adversarial bandit setting [Arora et al., 2012], and we have to make additional assumptions about the shape of the reward functions $f_{i}$. In this work, we assume that the underlying reward functions are initially increasing and concave, then decreasing.

Definition 1 (Single-peaked bandit). We call $f_{i}($.$) a single-$ peaked reward function, if there exists a tipping points $\bar{m}_{i}$ such that $f_{i}(m)$ increases monotonically in $m$ and is concave up to $m \leq \bar{m}_{i}$, and then decreases monotonically for $m>$ $\bar{m}_{i}$. We call a bandit with single-peaked reward functions a single-peaked bandit.

Note that bandits with monotonically increasing or decreasing reward functions are single-peaked bandits with $\bar{m}_{i}=\infty$ and $\bar{m}_{i}=0$, respectively.

\section{SPO: A New No-Policy-Regret Algorithm}

Our algorithm operationalizes the principle of optimism in the face of uncertainty, which has been successfully applied with different interpretations to a wide range of MAB problems [Bubeck et al., 2012]. Our interpretation of the principle is as follows: At each time step, pull the arm with the highest optimistic future reward. The reward functions of a singlepeaked bandit are first increasing and concave, then become decreasing. Therefore, we can define the future optimistic reward in the increasing phase using concavity and in the decreasing phase using monotonicity of the reward function. In the increasing concave phase, we estimate the optimistic future reward as

$$
p_{i}^{T}\left(n_{i}, t\right)=\sum_{s=t+1}^{T} \min \left\{1,\left(f_{i}\left(n_{i}\right)+\Delta_{i}\left(n_{i}\right) \cdot(s-t)\right)\right\},
$$

where $\Delta_{i}\left(n_{i}\right)=f_{i}\left(n_{i}\right)-f_{i}\left(n_{i}-1\right)$. Defined this way, $p_{i}^{T}\left(n_{i}, t\right)$ is a linear optimistic approximation of future rewards from arm $i$ after it has been pulled $n_{i}$ times within the first $t$ pulls. Similarly, for the decreasing phase, we can define

$$
p_{i}^{T}\left(n_{i}, t\right)=f_{i}\left(n_{i}\right) \cdot(T-t)
$$

In the increasing phase, we use the fact that the reward will increase at most linearly, and in the decreasing phase we use that it will at best remain constant.

The Single-Peaked Optimism algorithm (SPO, Algorithm 1) performs two main steps at every round $t$ :

1. Pull the arm that maximizes $p_{i}^{T}\left(n_{i}, t\right)$ where $n_{i}$ is the number of times the algorithm has pulled arm $i$ so far.

2. Update the optimistic future rewards $p_{i}^{T}\left(n_{i}, t\right)$.

For technical reasons, we add an initial phase in which we pull each $\operatorname{arm} \log (T)$ times, which only adds sub-linear policy regret, but simplifies the analysis (see Appendix C).

Our analysis formalizes the observation that while SPO may initially overestimate the future reward of an arm that grows at a high rate, it will stop pulling that arm as soon as it ceases to live up to the optimistic expectations.

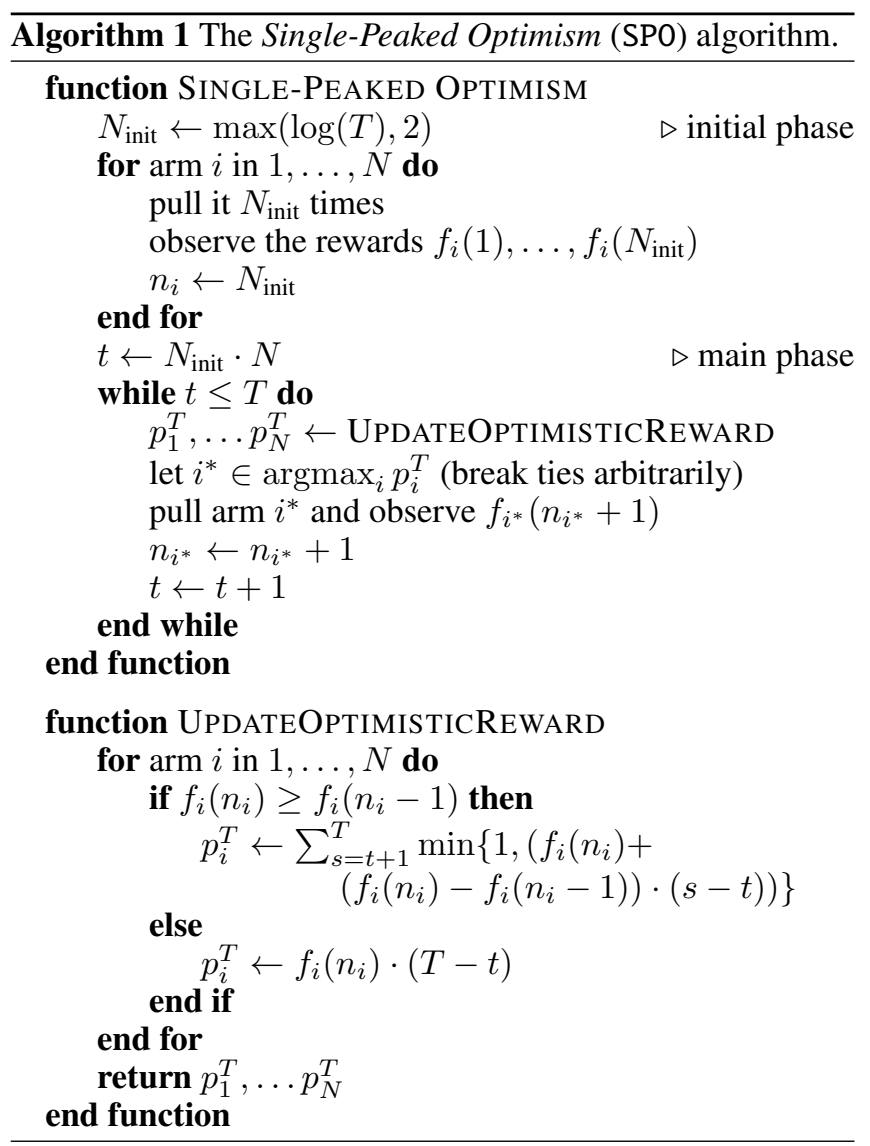

\subsection{Regret Analysis}

Next, we present our main theoretical result, which establishes the sub-linear policy regret of SPO. All omitted proofs and technical material can be found in Appendix C.

Theorem 1. [informal statement] For any (noise-free) single-peaked bandit, SPO achieves sub-linear policy regret.

The proof consists of several steps: we first observe that all single-peaked reward functions have finite asymptotes (Lemma 3, Appendix C), which follows from the monotone convergence theorem. Then, we show that for sufficiently large time horizons, always pulling the single arm with the highest asymptote would lead to sub-linear policy regret (Lemma 4, Appendix C). Finally, the key step of the proof is to show that SPO pulls all arms with suboptimal asymptotes less than linear in $T$. Together these steps imply the sub-linear policy regret of SPO.

\section{An LP-based Heuristics to Handle Noise}

So far we have assumed the decision-maker can observe rewards free of noise. In this section, we describe how to find an upper bound on the future reward from noisy observations. This allows us to extend SPO to noisy observation.

Assume that when pulling arm $i$ for the $n$-th time, we observe $\hat{f}_{i}(n)=f_{i}(n)+\varepsilon_{i}(n)$ where $\varepsilon_{i}$ is a random noise term. We start by assuming that the magnitude of the noise is bounded $\left|\varepsilon_{i}(n)\right| \leq \bar{\varepsilon}_{i}$, and $\bar{\varepsilon}_{i}$ is known for each arm. We 

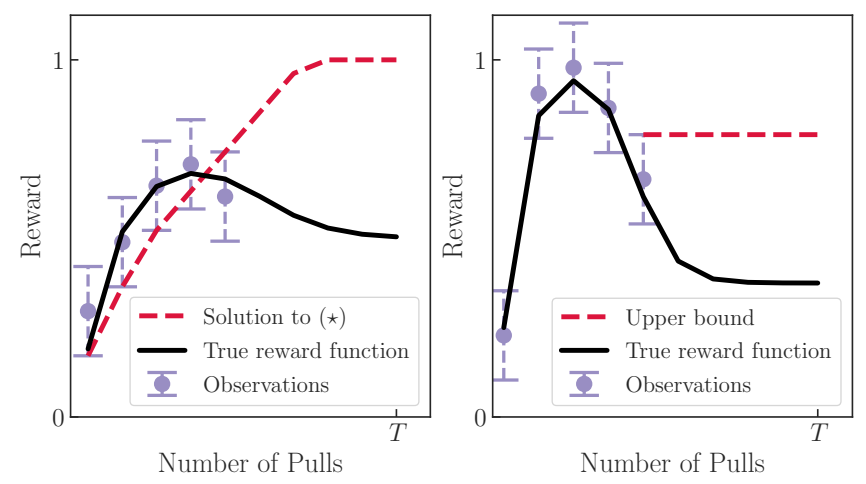

Figure 2: An illustration of 5 noisy reward observations from an arm, along with the true reward values which lie within the depicted confidence intervals. The dashed red curves specify our upper bound on cumulative future rewards obtained by solving $(\star)$. The left plot shows an instance in which $(\star)$ has a feasible concave and increasing solution. Note that the upper-bound estimate can be lower than the true reward function for past observation, but it is indeed an upper bound for future observations. The right plot shows an instance in which $(\star)$ is not feasible because the reward curve has entered its decreasing phase. In this case the last observation provide an upper bound on the cumulative future reward.

can then define $L_{i}^{j}=\hat{f}_{i}(n)-\bar{\varepsilon}_{i}$ and $U_{i}^{j}=\hat{f}_{i}(n)+\bar{\varepsilon}_{i}$ to obtain confidence intervals for the true reward $f_{i}(n)$ such that $f_{i}(n) \in\left[L_{i}^{j}, U_{i}^{j}\right]$ with probability 1.

We first extend our algorithm to this case of bounded noise, and then relax this assumption to confidence intervals that contain the true value with probability less than 1 .

\subsection{Decreasing Phase}

For arms in their decreasing phase, we can directly define the optimistic future return as $p_{i}^{T}\left(n_{i}, t\right)=U_{i}^{j} \cdot(T-t)$ using the confidence interval $\left[L_{i}^{j}, U_{i}^{j}\right]$.

\subsection{Increasing Phase}

For arms in their increasing phase, we can combine the confidence intervals with our knowledge that the function is concave. Concretely, we find the monotone concave function with the highest cumulative future reward that can explain past observations. We can phrase this as solving the following linear program (LP) for each arm $i$ :

$$
\begin{array}{lll}
\operatorname{maximize}_{v} & \sum_{j=n+1}^{n+T-t} v_{j} & \\
\text { subject to } & 0 \leq v_{j} \leq 1, & j=1, \ldots, T \\
& L_{i}^{j} \leq v_{j} \leq U_{i}^{j}, & j=1, \ldots, n \\
& v_{j} \leq v_{j+1}, & j=1, \ldots, T-1 \\
& v_{j} \leq 2 v_{j-1}-v_{j-2}, & j=3, \ldots, T
\end{array}
$$

where $n$ is the number of times arm $i$ has been pulled up to time $t$. The optimization variables $v_{j}$ correspond to the values of the reward function $f_{i}$ after $j$ pulls of arm $i$. The constraints encode that the true reward function is bounded, consistent with past observations, increasing, and concave, in that order. Hence, a feasible solution to the LP corresponds to a possible reward function and an optimal solution provides a tight upper bound on future rewards.

Theorem 2. Let $f_{i}: \mathbb{N}^{+} \rightarrow[0,1]$ be a concave, increasing function with confidence bounds $L_{i}^{1}, U_{i}^{1}, \ldots, L_{i}^{n}, U_{i}^{n} \in[0,1]$ such that $f_{i}(j) \in\left[L_{i}^{j}, U_{i}^{j}\right]$ for $1 \leq j \leq n$. Let $V^{*}=$ $\sum_{j=n+1}^{n+T-t} v_{j}$ be the solution to $(\star)$. Then, $\sum_{j=n+1}^{n+T-t} f_{i}(j) \leq$ $V^{*}$. Furthermore, there exists a concave, increasing function, $f_{i}^{*}: \mathbb{N}^{+} \rightarrow[0,1]$, such that $\sum_{j=n+1}^{n+T-t} f_{i}^{*}(j)=V^{*}$.

We can extend SPO to noisy observations, by solving the LP $(\star)$ every time we update the future optimistic reward for a given arm. If the LP does not have a feasible solution, we can infer that the arm is in its decreasing phase, and use a corresponding upper bound. Figure 2 illustrates both cases.

\subsection{Unbounded Noise}

We can readily extend this approach to unbounded noise with confidence intervals.

Corollary 1. Let $f_{i}: \mathbb{N}^{+} \rightarrow[0,1]$ be a concave, increasing function. Suppose that for any $\delta>0$ and observation $\hat{f}_{i}\left(n_{i}\right)$ we can find a confidence interval $\left[L_{i}^{n_{i}}(\delta), U_{i}^{n_{i}}(\delta)\right]$ such that $f_{i}\left(n_{i}\right) \in\left[L_{i}^{n_{i}}(\delta), U_{i}^{n_{i}}(\delta)\right]$ with probability at least $1-\delta$. Let $V^{*}$ be the solution to $(\star)$. Then for any $\epsilon>0$, we can choose $\delta$ such that $\sum_{j=n+1}^{n+T-t} f_{i}(j) \leq V^{*}$ with probability at least $1-\epsilon$.

The proof sketch goes as follows: The probability that within the remainder of time horizon $T$, at least one true reward value falls outside of its confidence interval is upper bounded by $1-(1-\delta)^{T}$. For the given $\epsilon$, we can choose $\delta \leq 1-\mathrm{e}^{\frac{1}{T} \log (1-\epsilon)}$, so that the probability of any true reward being outside its confidence interval is bounded by $\epsilon$. More precisely, we can write:

$$
\begin{aligned}
1-(1-\delta)^{T} & \leq 1-\left(1-\left(1-\mathrm{e}^{\frac{1}{T} \log (1-\epsilon)}\right)\right)^{T} \\
& =1-\mathrm{e}^{\log (1-\epsilon)}=\epsilon
\end{aligned}
$$

With the above choice for $\delta$, the optimistic future reward is estimated correctly with probability at least $1-\epsilon$.

\section{Experiments}

In this section, we empirically investigate the effectiveness of our noise-handling approach on several datasets. ${ }^{5}$

Setup. We consider three datasets: (1) a set of synthetic reward functions, (2) a simulation of a user interacting with a recommender system, and (3) a dataset constructed from the FICO credit scoring data. We compare SPO with six baselines: (1) a greedy algorithm that always pulls the arm that provided the highest reward at the last pull, (2) a onestep-optimistic variant of SPO that pulls the arm with the highest upper bound on the reward at the next pull, (3) EXP3, a standard no-external-regret algorithm for adversarial bandits [Auer et al., 2002], (4) R-EXP3, a modification of EXP3 for non-stationary bandits [Besbes et al., 2019],

\footnotetext{
${ }^{5}$ Code to reproduce all of our experiments can be found at https://github.com/david-lindner/single-peaked-bandits.
} 

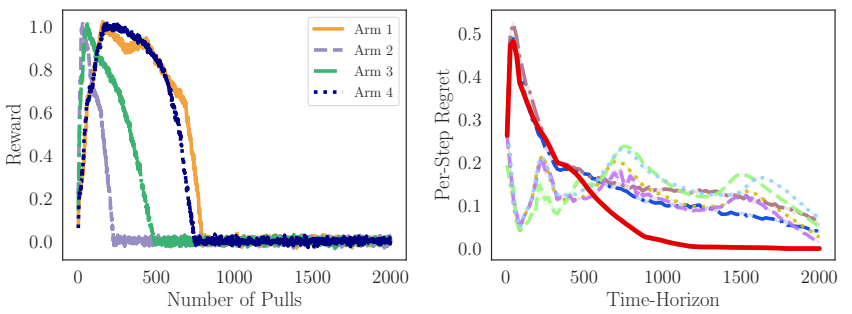

(a) FICO Dataset
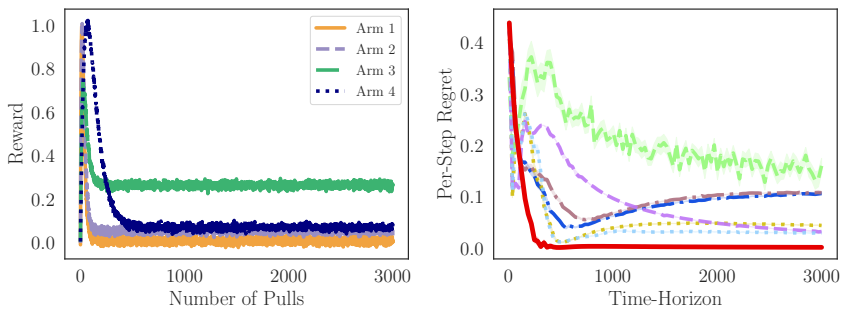

(b) Recommender System Simulations

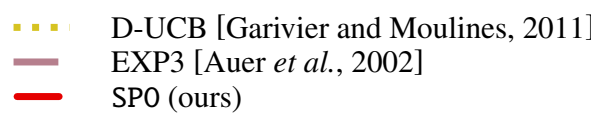

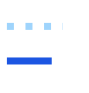

SW-UCB [Garivier and Moulines
R-EXP3 [Besbes et al., 2019] $\begin{array}{ll}-= & \text { Greedy } \\ -= & \text { One-Step-Optimistic }\end{array}$

Figure 3: Results of our simulation experiments with (a) the FICO credit scoring dataset, and (b) synthetic recommender system data. In both cases, the left plot shows the reward functions of the bandit, and the right plot shows the per-step regret, i.e., the policy regret divided by $T$. The $\mathrm{x}$-axes of the regret plots show the time horizon $T$, discretized in 100 points, where each point corresponds to a single experiment. The per-step regret is averaged over 30 random seeds. SPO outperforms all baselines and is the only algorithm that achieves low policy regret for long time horizons.

(5) discounted UCB (D-UCB), and (6) sliding window UCB (SW-UCB), two adaptations of UCB to nonstationary bandits [Garivier and Moulines, 2011].

Illustrations on synthetic data. We first perform a series of experiments on single-peaked bandits with two arms, and synthetic reward functions with Gaussian noise. To this end we define a class of single-peaked functions and combine them into multiple single-peaked bandits with two arms each. In Figure 1 we highlight one experiment in which algorithms that minimize external regret fail. The figure shows how SPO avoids this kind of failure by minimizing policy regret. In Appendix D we provide more detailed results comparing SPO to the baselines on various synthetic reward functions, including the monotonic functions proposed by [Heidari et al., 2016], and evaluate the effect of varying the observation noise. We find that SPO matches the performance of the baselines in all cases and significantly outperforms them in some. Further, we show that SPO can also handle stationary MABs, where arms have fixed reward distributions.

FICO credit lending data. Motivated by our initial example of a budget planner in Section 1, we simulate a credit lending scenario based on the FICO credit scoring dataset from 2003 [Reserve, 2007]. We pre-process the data using code provided by previous work [Hardt et al., 2016; Liu et al., 2018]. The dataset contains four ethnic groups: 'Asian', 'Black', 'Hispanic' and 'White'. Each group has a distribution of credit-scores and an estimated mapping from credit-scores to the probability of repaying a loan. We use this group-level data to simulate a population of individuals applying for a loan. Each individual belongs to one ethnic group and has a credit score sampled from the group's distribution and a probability of repaying a loan.

We consider a hypothetical decision-making scenario in which at each round, there is exactly one loan applicants from each group. In each time step, the decision-maker (i.e., a bank) can approve only one loan applicant. We are interested in the long-term impact of decision-maker's choices on the underlying groups. As discussed in Section 1, we argue that a fair decision-maker will allocate loans according to the groups' long-term potential to turn them into welfare/prosperity, which is measured by the per-group reward functions in this simulation. In other words, policy regret is our measure of long-term disparity and achieving low policy regret improves the fairness of resource allocation decisions.

To simulate this situation based on the FICO dataset, we first sample $N$ applicants from each group. We assume the decision-maker always approves the loan of the applicant with the highest credit score within a given group; hence, we order the applicants decreasing by their credit score. Thereby, we reduce the problem to the decision-maker deciding between four arms to pull, each corresponding to one group. We interpret pulling an arm as approving the load on the highest scoring applicant within the corresponding group. However, the credit scores do not directly correspond to the reward of pulling an arm. Rather we want to define a reward function that quantifies the benefit/loss of giving out a loan to a group. ${ }^{6}$ We follow [Liu et al., 2018], and measure the impact on a group as the change in mean credit score for this group. Liu et al.'s model assumes an increase in credit score of 75 points for a repaid loan and a decrease of 150 points for a defaulted loan, while the credit score is always being clipped to the range $[300,850]$. Finally, we rescale the rewards to $[0,1]$.

The resulting reward functions increase at first because the first individuals in each group are highly credit-worthy and them paying back their loan increases the mean credit score for the group. The reward functions are concave because as the decision-maker gives more loans to a group he/she starts to give loans to less creditworthy individuals. Eventually, the reward functions start to decrease because giving loans to individuals who cannot pay them back decreases the average credit score of the group. Hence, this setup can be approximately modelled as a single-peaked bandit.

Figure 3a shows results of running SPO in this setup. Overall, SPO strictly outperforms the baselines over long time hori-

\footnotetext{
${ }^{6}$ We also investigated a variant of this setting considering only the utility of a loan to the decision-maker, see Appendix D.
} 
zons. For short time horizons we find that simple greedy approaches or UCB variants can perform favorably.

Synthetic recommender system data. Recent work shows that strategies minimizing external regret can perform poorly in the context of recommender systems, due to negative feedback loops [Jiang et al., 2019; Warlop et al., 2018; Mladenov et al., 2020; Chaney et al., 2018]. Here, we focus on one concrete problem that can arise: many recommender systems exhibit a bias towards recommending particularly engaging or novel content that leads to high instantaneous reward, disregarding the long-term benefit and cost to the user. We argue that this situation is analogous to our example of a budget maker, and that a recommender system should aim to maximize it's users' long-term benefit.

Motivated by this observation, we simulate a system that recommends content, e.g., articles or videos, to a user and receives feedback about how much the user engaged with the content. For simplicity, we assume the user's engagement with a piece of content is driven by two factors only: (i) the user's inherent preferences, and (ii) a novelty factor which makes new content more engaging to the user. We assume that the user's inherent preferences stay constant, but the novelty factor decays when showing an item more often. Note that an algorithm that minimizes external regret would show content with high novelty and neglect content that is a better match for the user's inherent preferences. An algorithm that minimizes policy regret would select the content that best matches the user's inherent preferences in the long-run.

We simulate the user's feedback with a reward function $f_{i}$ for each item that can be recommended. Each item has an inherent value $v$ to the user, a novelty factor $n$, and decay factors $\gamma$ and $c$. The reward is $f_{i}(0)=0$ for never showing an item, and subsequent rewards are defined as

$$
f_{i}(t)=f_{i}(t-1)+n \cdot \gamma^{t}-c \cdot\left(f_{i}(t)-v\right) .
$$

The second term in the expression models the novelty of an item which decays when showing it more often. The third term models the tendency of the reward to move towards how much the user values the item inherently. The resulting rewards increase at first due to the novelty of an item and decrease later as the novelty factor decays. For simplicity we model all effects that are not captured by this stylized model as Gaussian noise on the observed rewards.

Figure $3 b$ shows that SPO significantly outperforms the baselines for long time horizons, at the cost of worse performance for short time horizons. This results indicates that if a decision-maker acts on a short time-horizon classical bandit algorithms perform well. However, if the decisionmaker aims to achieve a good long-term impact, SPO is preferable. We present results on additional instances of the recommender system simulation in Appendix D.

\section{Conclusion}

Motivated by several real-world domains, we studied single-peaked bandits in which the reward from each arm is initially increasing then decreasing in the number of pulls of the arm. We introduced Single-Peaked Optimism (SPO), an algorithm that achieves sub-linear policy regret in single-peaked bandits. Our findings highlight the importance of understanding the long-term implications of ML-based decisions for impacted communities and society at large, and utilizing domain knowledge, e.g., regarding social- and population-level dynamics stemming from decisions today, to design appropriate sequences of allocations that do not amplify historical disparities.

Limitations. We argued that single-peaked bandits are a useful model to provide insights about allocation decisions in a range of practical domains, e.g., allocating loans to communities, allocating funds to research institutions, or allocating policing resources to districts. However, single-peaked bandits can also be too restrictive in domains where the evolution of rewards are more nuanced, e.g., if rewards can later increase again after first decreasing. We emphasize that singlepeaked reward functions are one among many reasonable classes of reward functions that are interesting to study from an algorithmic perspective. We consider our work as an starting point to look into more complex dynamics in future work.

Future work. We hope that our work draws the research community's attention to the study of policy regret for typical reward-evolution curves. Additional directions for future work include (1) establishing regret bounds for settings with arbitrary noise distributions, (2) providing instance-specific (and potentially tighter) regret bounds for Single-Peaked Optimism, and finally (3) more broadly characterizing the limits of "optimism in the face of uncertainty" principle in achieving low policy regret.

\section{Acknowledgements}

Lindner was partially supported by Microsoft Swiss JRC. This work was in part done while Heidari was a postdoctoral fellow at ETH Zurich. Heidari acknowledges partial support from NSF IIS2040929. Any opinions, findings, and conclusions, or recommendations expressed in this material are those of the authors and do not necessarily reflect the views of the National Science Foundation, or other funding agencies.

\section{References}

[Angwin et al., 2016] Julia Angwin, Jeff Larson, Surya Mattu, and Lauren Kirchner. Machine bias. Propublica, 2016.

[Arora et al., 2012] Raman Arora, Ofer Dekel, and Ambuj Tewari. Online bandit learning against an adaptive adversary: from regret to policy regret. In ICML, 2012.

[Auer et al., 2002] Peter Auer, Nicolo Cesa-Bianchi, Yoav Freund, and Robert E Schapire. The nonstochastic multiarmed bandit problem. SIAM journal on computing, 32(1):48-77, 2002.

[Berk and Hyatt, 2015] Richard Berk and Jordan Hyatt. Machine learning forecasts of risk to inform sentencing decisions. Federal Sentencing Reporter, 27(4):222-228, 2015.

[Besbes et al., 2019] Omar Besbes, Yonatan Gur, and Assaf Zeevi. Optimal exploration-exploitation in a multi-armed bandit problem with non-stationary rewards. Stochastic Systems, 9(4):319$337,2019$.

[Bubeck et al., 2012] Sébastien Bubeck, Nicolo Cesa-Bianchi, et al. Regret analysis of stochastic and nonstochastic multi-armed bandit problems. Foundations and Trends ${ }^{\circledR}$ in Machine Learning, 5(1):1-122, 2012. 
[Buolamwini and Gebru, 2018] Joy Buolamwini and Timnit Gebru. Gender shades: Intersectional accuracy disparities in commercial gender classification. In Conference on Fairness, Accountability, and Transparency, 2018.

[Chaney et al., 2018] Allison JB Chaney, Brandon M Stewart, and Barbara E Engelhardt. How algorithmic confounding in recommendation systems increases homogeneity and decreases utility. In Conference on Recommender Systems, 2018.

[Combes and Proutiere, 2014] Richard Combes and Alexandre Proutiere. Unimodal bandits: Regret lower bounds and optimal algorithms. In ICML, 2014.

[Dobbie et al., 2018] Will Dobbie, Andres Liberman, Daniel Paravisini, and Vikram Pathania. Measuring bias in consumer lending. Technical report, National Bureau of Economic Research, 2018.

[Dong et al., 2018] Jinshuo Dong, Aaron Roth, Zachary Schutzman, Bo Waggoner, and Zhiwei Steven Wu. Strategic classification from revealed preferences. In Conference on Economics and Computation, 2018.

[Dwork et al., 2012] Cynthia Dwork, Moritz Hardt, Toniann Pitassi, Omer Reingold, and Richard Zemel. Fairness through awareness. In ITCS, 2012.

[Ensign et al., 2017] Danielle Ensign, Sorelle A. Friedler, Scott Neville, Carlos Eduardo Scheidegger, and Suresh Venkatasubramanian. Decision making with limited feedback: Error bounds for predictive policing and recidivism prediction. In Conference on Fairness, Accountability, and Transparency, 2017.

[Ensign et al., 2018] Danielle Ensign, Sorelle A. Friedler, Scott Neville, Carlos Eduardo Scheidegger, and Suresh Venkatasubramanian. Runaway feedback loops in predictive policing. In Conference on Fairness, Accountability, and Transparency, 2018.

[Garivier and Moulines, 2011] Aurélien Garivier and Eric Moulines. On upper-confidence bound policies for switching bandit problems. In International Conference on Algorithmic Learning Theory, 2011.

[Gittins et al., 2011] John Gittins, Kevin Glazebrook, and Richard Weber. Multi-armed bandit allocation indices. John Wiley \& Sons, 2011.

[Hardt et al., 2016] Moritz Hardt, Eric Price, and Nati Srebro. Equality of opportunity in supervised learning. In NeurIPS, 2016.

[Heidari et al., 2016] Hoda Heidari, Michael J. Kearns, and Aaron Roth. Tight policy regret bounds for improving and decaying bandits. In IJCAI, 2016.

[Heidari et al., 2019] Hoda Heidari, Vedant Nanda, and Krishna P Gummadi. On the long-term impact of algorithmic decision policies: Effort unfairness and feature segregation through social learning. In ICML, 2019.

[Hu and Chen, 2018] Lily $\mathrm{Hu}$ and Yiling Chen. A short-term intervention for long-term fairness in the labor market. In $W W W$, 2018.

[Hu et al., 2019] Lily Hu, Nicole Immorlica, and Jennifer Wortman Vaughan. The disparate effects of strategic manipulation. In Conference on Fairness, Accountability, and Transparency, 2019.

[Jabbari et al., 2017] Shahin Jabbari, Matthew Joseph, Michael Kearns, Jamie Morgenstern, and Aaron Roth. Fairness in reinforcement learning. In ICML, 2017.

[Jiang et al., 2019] Ray Jiang, Silvia Chiappa, Tor Lattimore, András György, and Pushmeet Kohli. Degenerate feedback loops in recommender systems. In Conference on AI, Ethics, and Society, 2019.

[Joseph et al., 2016] Matthew Joseph, Michael Kearns, Jamie Morgenstern, and Aaron Roth. Fairness in learning: Classic and contextual bandits. In NeurIPS, 2016.

[Joseph et al., 2017] Matthew Joseph, Michael Kearns, Jamie Morgenstern, Seth Neel, and Aaron Roth. Fair algorithms for infinite contextual bandits. In Conference on Fairness, Accountability, and Transparency, 2017.
[Kannan et al., 2019] Sampath Kannan, Aaron Roth, and Juba Ziani. Downstream effects of affirmative action. In Conference on Fairness, Accountability, and Transparency, 2019.

[Kleinberg and Immorlica, 2018] Robert Kleinberg and Nicole Immorlica. Recharging bandits. In Annual Symposium on Foundations of Computer Science (FOCS), pages 309-319, 2018.

[Kleinberg et al., 2017] Jon Kleinberg, Sendhil Mullainathan, and Manish Raghavan. Inherent trade-offs in the fair determination of risk scores. In ITCS, 2017.

[Levine et al., 2017] Nir Levine, Koby Crammer, and Shie Mannor. Rotting bandits. In NeurIPS, 2017.

[Liu et al., 2018] Lydia T Liu, Sarah Dean, Esther Rolf, Max Simchowitz, and Moritz Hardt. Delayed impact of fair machine learning. In ICML, 2018.

[Marcinkowski et al., 2020] Frank Marcinkowski, Kimon Kieslich, Christopher Starke, and Marco Lünich. Implications of AI (un-) fairness in higher education admissions: the effects of perceived AI (un-) fairness on exit, voice and organizational reputation. In Conference on Fairness, Accountability, and Transparency, 2020.

[Milli et al., 2019] Smitha Milli, John Miller, Anca D Dragan, and Moritz Hardt. The social cost of strategic classification. In Conference on Fairness, Accountability, and Transparency, 2019.

[Mladenov et al., 2020] Martin Mladenov, Elliot Creager, Omer Ben-Porat, Kevin Swersky, Richard Zemel, and Craig Boutilier. Optimizing long-term social welfare in recommender systems: A constrained matching approach. In ICML, 2020.

[Moulin, 2004] Hervé Moulin. Fair division and collective welfare. MIT press, 2004.

[Mouzannar et al., 2019] Hussein Mouzannar, Mesrob I Ohannessian, and Nathan Srebro. From fair decision making to social equality. In Conference on Fairness, Accountability, and Transparency, 2019.

[Reserve, 2007] US Federal Reserve. Report to the congress on credit scoring and its effects on the availability and affordability of credit. Board of Governors of the Federal Reserve System, 2007.

[Robbins, 1952] Herbert Robbins. Some aspects of the sequential design of experiments. Bulletin of the American Mathematical Society, 58(5):527-535, 1952.

[Sánchez-Monedero et al., 2020] Javier Sánchez-Monedero, Lina Dencik, and Lilian Edwards. What does it mean to 'solve' the problem of discrimination in hiring? social, technical and legal perspectives from the uk on automated hiring systems. In Conference on Fairness, Accountability, and Transparency, 2020.

[Sandel, 2010] Michael J Sandel. Justice: What's the right thing to do? Macmillan, 2010.

[Sweeney, 2013] Latanya Sweeney. Discrimination in online ad delivery. Queue, 11(3):10, 2013.

[Tekin and Liu, 2012] Cem Tekin and Mingyan Liu. Online learning of rested and restless bandits. Transactions on Information Theory, 58(8):5588-5611, 2012.

[Warlop et al., 2018] Romain Warlop, Alessandro Lazaric, and Jérémie Mary. Fighting boredom in recommender systems with linear reinforcement learning. In NeurIPS, 2018.

[Whittle, 1988] Peter Whittle. Restless bandits: Activity allocation in a changing world. Journal of applied probability, pages 287298, 1988 .

[Yu and Mannor, 2011] Jia Yuan Yu and Shie Mannor. Unimodal bandits. In $I C M L, 2011$.

[Zafar et al., 2017] Muhammad Bilal Zafar, Isabel Valera, Manuel Gomez Rodriguez, and Krishna P. Gummadi. Fairness beyond disparate treatment \& disparate impact: Learning classification without disparate mistreatment. In $W W W, 2017$. 